\title{
Underwater Acoustic Noise Measurement in Test Tanks
}

\author{
N. Cochard, J. L. Lacoume, P. Arzeliès, and Y. Gabillet
}

\begin{abstract}
The range capability of underwater acoustic equipment installed onboard underwater vehicles is limited by the noise generated by propellers, hydraulic pumps .... Measuring this noise at sea is quite expensive. Here is described a procedure allowing the measurement of the radiated noise in test tanks. This method is derived from techniques previously developed in aerial acoustics and in electromagnetism.
\end{abstract}

Index Terms-Noise level measurement, reverberation, underwater acoustics.

\section{INTRODUCTION}

$\mathrm{T}$ HE measurement of the level of underwater acoustic noise sources is generally made in two principal ways. The most direct way consists of measurements in the open sea. This operation is expensive in time and in operation means. Noise measurement of a source in a test tank is a suitable alternative allowing developement of easy measurements.

Level measurement methods exist in an enclosed medium in aerial acoustics [7], [8], for electromagnetic sources [4] and in underwater acoustics [6]. The sound intensity technique developed in aerial acoustics can be applied to underwater acoustics for the measurement of a source power in test tanks [5, pp. 198]. Some results in underwater acoustics are presented in [3]. Hovewer, this technique cannot succeed when measuring propeller noise. In this measurement the propeller gives rise to water currents. It is shown in [2] that, in this situation, the measument has to take into account the speed of the currents. This shows that this technique would be very difficult, if not impossible, to implement for the measurement of propeller noise.

Here we introduce an operational method for the measurement of underwater acoustic noise sources level in test tanks. We give the basic principles of the measurement and the experimental procedure. Then we present the results obtained with wideband sources and pure frequencies and we characterize the precision experimentally.

The paper is organized as follows. In Section II, we give the principles of the method. Section III describes the methodology. In Section IV we present experimental results obtained with a calibrated source and with an operational device.

Manuscript received October 23, 1998; revised April 6, 2000.

N. Cochard and P. Arzeliès are with the IFREMER Centre de Toulon-La Seyne, La Seyne sur Mer cedex, France.

J. L. Lacoume is with the LIS UMR 38402 Saint Martin d'Hères, France.

Y. Gabillet, deceased, was with the CSTB 38400 Saint-Martin d'Hères, France.

Publisher Item Identifier S 0364-9059(00)09289-X.

\section{MEASUREMENT METHOD}

When an acoustic source emits in a test tank, acoustic waves are reflected by the walls, the surface, and the bottom. The multiple reflections give rise to a reverberated field. The balance is reached when the losses issuing from reflections on the walls compensate the acoustic power driven by the source. Then, the acoustic energy in the tank depends on the power of the source and on the losses on the walls. In order to get the power of the source, we have to measure the acoustic energy in the reverberated field and to determine the relation between this energy and the source power.

\section{A. The Acoustic Field in the Tank}

Following the classical approach developed in aerial acoustics [7], [8] we assume that the acoustic field in the tank contains two parts: the direct field, with an acoustic energy density $W_{d}$ identical to the source acoustic energy density in an open medium, and the reverberated field, with an acoustic energy density $W_{r}$ issuing from the multiple reflections on the walls.

1) The Direct Field: The acoustic energy density of the direct field depends on the distance $r$ to the source by

$$
W_{l l}(r)=\frac{P_{s}}{4 \pi c r^{2}}
$$

where $P_{s}$ is the power of the source and $c$ the sound velocity.

This assumes an omnidirectional source. If the source is directional the direct field depends on the direction. The method presented here cannot determine the directional properties of the source. In this case we associate to the source an omnidirectional one which emits the same acoustic power.

2) The Reverberated Field: The reverberated field is assumed homogeneous in space and isotropic [8], [10]. The homogeneity and the isotropy of the reverberated field depend on the transmitted signal and on the measurement bandwidth. The spatial homogeneity of the reverberated field will be checked using measurements taken in different locations. The case of pure frequency tones that do not fullfill the condition of mode mixing will also be considered.

For an isotropic homogeneous reverberated field the acoustic energy density $W_{r}$ and the acoustic intensity $I_{r}$ are related by [7], [8], [10]

$$
W_{r}=\frac{4 I_{r}}{c}
$$

The time derivative of the total acoustic power contained in the tank, $Q=W_{r} V$ ( $V$ beeing the volume of the water), is the difference between the acoustic power driven by the source $P_{s}$ 
and the acoustic power dissipated on the walls by absorption or transmission $a I_{r}$

$$
\frac{d Q}{d t}=P_{s}-a I_{v}=P_{s}-\frac{a c}{4 V} Q
$$

In this relation $a$ is the Sabine coefficient [11] that characterizes the absorption and the transmission by the walls.

When the balance is reached, $(d Q / d t)=0$ and

$$
P_{s}=\frac{a c}{4 V} Q=\frac{a c}{4} W_{r}
$$

This is the relation between the source power $\left(P_{s}\right)$ and the acoustic energy density in the reverberate field $\left(W_{T}\right)$.

In order to relate these two quantities, we must know the Sabine coefficient $a$. This coefficient is given by the reverberation time.

\section{B. The Reverberation Time}

A stationary source giving a total acoustic energy in the tank $Q_{0}$ is switched off at time $t=0$. For positive time the acoustic power is

$$
Q(t)=Q_{0} e^{-\frac{a c}{4 t} t}, \quad t>0
$$

The reverberation time $T_{r}$ is the duration that the acoustic energy of the reverberated field takes to decrease $60 \mathrm{~dB}$ [8]

$$
T_{r}=\frac{24}{\log e} \frac{V}{a c}=55.3 \frac{V}{a c}
$$

from the reverberation time we get the Sabine coefficient

$$
a=\frac{24}{\log e} \frac{V}{T_{r} c}=55.3 \frac{V}{T_{T} c} .
$$

In order to measure the Sabine coefficient, we have to localize the direct field and the reverberated field in the tank.

\section{The Direct Field Zone}

As in [7] we propose to define the direct field zone as the part of the tank where the acoustic energy clensity of the direct field $W_{d}(r)$ is greater than the acoustic energy density of the reverberated field $W_{r}$, minus $3 \mathrm{~dB}$.

From (1) and (4)

$$
\frac{W_{r}}{W_{d}(r)}=\frac{16 \pi r^{2}}{a}
$$

Thus the direct field zone is the sphere of radius

$$
r_{l}=\left(\frac{10^{0.3} a}{16 \pi}\right)^{1 / 2}=0.2 \sqrt{a} .
$$

It is important to verify, a key point for the applicability of the method, that this value does not exceed the dimensions of the tank. We will see later that this condition is fullfilled in the situations considered.

\section{Spectral Level of the Source}

The spectral level of a source emitting, in an open medium, a spectral pressure $p_{d}(f)$ at $1 \mathrm{~m}$ is [1]

$$
N_{s}(f)=20 \log \frac{p_{d}(f)}{p_{\mathrm{ref}}}
$$

where $p_{\text {ref }}$ is the reference spectral pressure.

For a source emitting in an open medium an acoustic spectral power $P_{s}(f)$, the spectral pressure $p_{d}(f)$ at $1 \mathrm{~m}$ is

$$
p_{d}^{2}(f)=\frac{\rho c}{4 \pi} P_{s}(f)
$$

where $\rho$ is the mass density of the water contained in the tank.

In the reverberated field, using (4)

$$
p_{\mathrm{rev}}^{2}(f)=\rho c^{2} W_{r}=\frac{4 \rho c}{a} P_{s}(f)
$$

Thus, using (5)

$$
\frac{p_{d}^{2}(f)}{p_{\mathrm{rev}}^{2}(f)}=\frac{a}{16 \pi}=\frac{55.3}{16 \pi} \frac{V}{T_{r} c}=1.1 \frac{V}{T_{r} c} .
$$

The spectral level [1] versus the spectral pressure $p_{\text {rev }}(f)$ in the reverberated field is

$$
N_{s}(f)=20 \log \frac{p_{\text {rev }}(f)}{p_{\text {ref }}}+10 \log \frac{V}{T_{r} c}+0.4 .
$$

This relation shows that, knowing the volume of water, we get the spectral level of the source from the measurement of reverberation time $T_{r}$ and of the spectral pressure in the reverberated field $p_{\text {rev }}(f)$.

\section{E. Homogeneity and Isotropy of the Reverberated Field}

This analysis is based on the homogeneity and the isotropy of the reverberated field. Considering acoustic modes, these conditions are fullfilled if a sufficient number of modes are excited in the tank giving a mode mixing [9]. The mode mixing condition [12] depends on the bandwidth and on the mean frequency separation of the modes.

1) Bandwidth of the Modes: The tank walls are not perfect reflectors, so the modes are attenuated. The reverberation time gives the attenuation. Each mode is a pure frequency tone attenuated by an exponential term. One mode at the resonant frequency $f_{r}$ is proportional to

$$
\exp \left(-3 t / T_{r} \log e\right) \cos \left(2 \pi f_{r} t+\varphi\right)
$$

A straightforward calculation gives then the 3 - $\mathrm{dB}$ bandwidth, $B_{3 \mathrm{~dB}}$, of the modes versus the reverberation time $T_{r}$.

$$
B_{3 \mathrm{~dB}}=\frac{6}{\pi \log e \cdot T_{r}}=\frac{4.4}{T_{r}} .
$$

2) Mean Frequency Separation of 2 Modes: In the three-dimensional $\left(k_{1}, k_{2}, k_{3}\right)$ wave number space, the modes are on a cubic grid. The volume of the wave number space cubes is

$$
k_{1} k_{2} k_{3}=\frac{(2 \pi)^{3}}{V}
$$

with $V$ being the volume of the tank. 
The modulus of the modes wave numbers in the frequency band $[f, f+\Delta f]$ are in the spherical shell of the wave number space

$$
2 \pi \frac{f}{c}<|\vec{k}|<2 \pi \frac{f+\Delta f}{c} .
$$

The volume of this spherical shell is $(2 \pi)^{3}\left(4 \pi f^{2} \Delta f / c^{3}\right)$.

The number of modes contained in the frequency band $[f, f+$ $\Delta f]$ is thus

$$
N_{f, \Delta f}=\frac{4 \pi f^{2} \Delta f V}{c^{3}}
$$

Giving the mean frequency separation between two modes

$$
\Delta f_{\text {mean }}=\frac{c^{3}}{4 \pi f^{2} V}
$$

3) The Mode Mixing Condition: In [12] Schroeder postulates that the reverberated field is isotropic and homogeneous if at least 20 modes are contained in the $3-\mathrm{dB}$ bandwidth of one mode. With the preceeding results this condition implies that

$$
20 \frac{c^{3}}{4 \pi f^{2} V} \leq \frac{6}{\pi \log e \cdot T_{r}}
$$

This condition is fullfilled if the frequency is greater than

$$
f_{\text {min }}=\left(\frac{5(\log e) c^{3} T_{r}}{6 V}\right)^{\frac{1}{2}}=0.6 \sqrt{\frac{c^{3} T_{r}}{V}} .
$$

In our case, $V=900 \mathrm{~m}^{3}$ and $T_{r}=0.4 \mathrm{~s}$ give $f_{\min }=740 \mathrm{~Hz}$. So the mode mixing condition is fullfilled in our band of interest $1 \mathrm{kHz}-20 \mathrm{kHz}$.

\section{F. Summary}

The preceeding results contain the principles of the measurement. With a sensor in the reverberated field, that is to say at a sufficient distance from the source, we determine the spectral pressure in the reverberated field $p_{\text {rev }}(f)$. This spectral pressure is related to the spectral level of the source by (9). This relation depends on the reverberation time that can be determined by a preliminary measurement. This model assumes that the reverberated field is homogeneous and isotropic. This condition is fullfilled when the mode mixing condition holds, that is to say when the frequency is greater than $f_{\text {min }}$ given by (10).

We now describe the measurement procedure.

\section{Measurement Procedure}

The measurement procedure is clivided in two steps. First, we put the source to be controlled in the tank. With a noise source switched off at $t=0$, we calibrate the tank by the determination of the reverberation time and of the direct field extension. Then, we activate the source under control and we measure the spectral pressure in one or in some randomly distributed positions in the reverberated field. Equation (9) then gives then the source level.

\section{A. Reverberation Time}

This measurement is made using a transducer of known characteristics as source and a hydrophone as receiver. The sensor output voltage $v(t)$ is proportional to the acoustic pressure at the point of measurement.

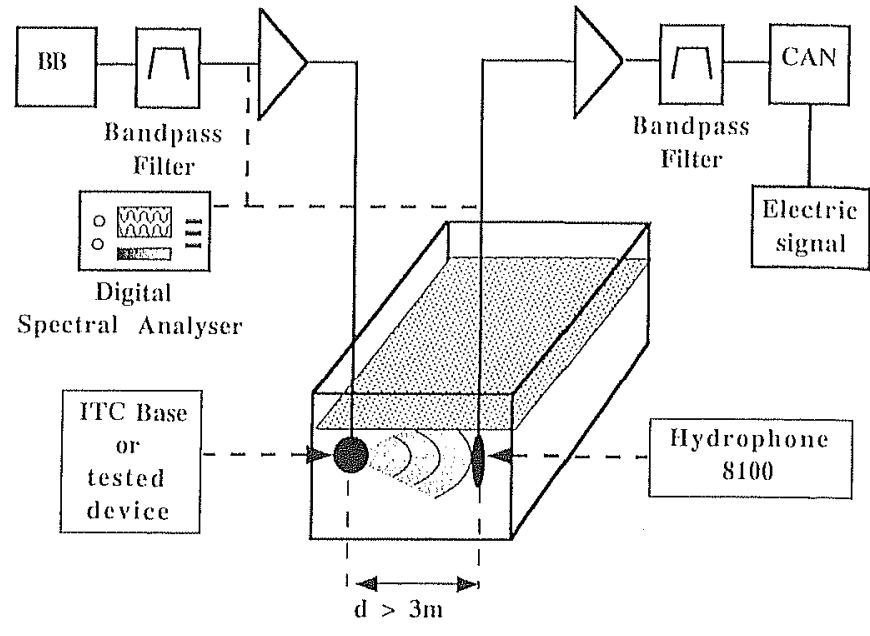

Fig. 1, The experimental setup.

The transducer is driven by a white noise covering the frequency band of interest. At time $t=0$ the transducer is swiched off and we measure the evolution of the hydrophone output voltage $v(t)$. In order to get the slope of the mean square value decrease we have to square $v(t)$ and to integrate. These operations are done numerically using the sampled output voltage $v(n)$ with a sampling frequency $\left(\nu_{E}\right)$ of $48 \mathrm{kHz}$. The integration is given by

$$
P(0)=v^{2}(0) \quad P(n)=v^{2}(n)+\alpha P(n-1), \quad n>0 .
$$

The impulse response of this integrator is

$$
h(n)=\alpha^{n}=e^{\left(n T_{E}\right) \nu_{E} \log \alpha}=e^{-\frac{n T_{E}}{T_{i}}} \text { for } n \geq 0,
$$

where $\left(T_{E}\right)$ is the sampling period and $\left(\nu_{E}\right)$ the sampling frequency. The time of integration: $T_{i}=-\left(1 / \nu_{E} \log \alpha\right)$ is choosen in order to average the signal and to not bias the decrease slope.

Then by a linear regression on the linear part of the plot of $P(n)$ we determine the reverberation time. In this measurement we have to be sure that the source power is wêll above the acoustic ambient noise present in the tank.

\section{B. Measurement of the Source Spectral Level}

We activate the source under control and we measure the spectral voltage given by the output of one or several hydrophones located in the reverberated field. The spectral voltage $v(f)$ is related to the spectral pressure $p_{\text {rev }}(f)$ through the receiving sensitivity of the hydrophone $S_{h}(\mathrm{~dB}$ refV $/ \mu \mathrm{Pa})$

$$
20 \log p_{\text {rev }}(f)=20 \log v(f)-S_{h} .
$$

From (9), the source spectral level for a reference spectral pressure of $1 \mu \mathrm{Pa} / \sqrt{\mathrm{Hz}}$ is

$$
N_{s}(f)=20 \log v(f)-S_{h}+10 \log \frac{V}{T_{r} c}+0.4 .
$$

\section{Accuracy}

The causes of uncertainties are:

a) the hypothesis of homogeneity and isotropy of the reverberated field; 


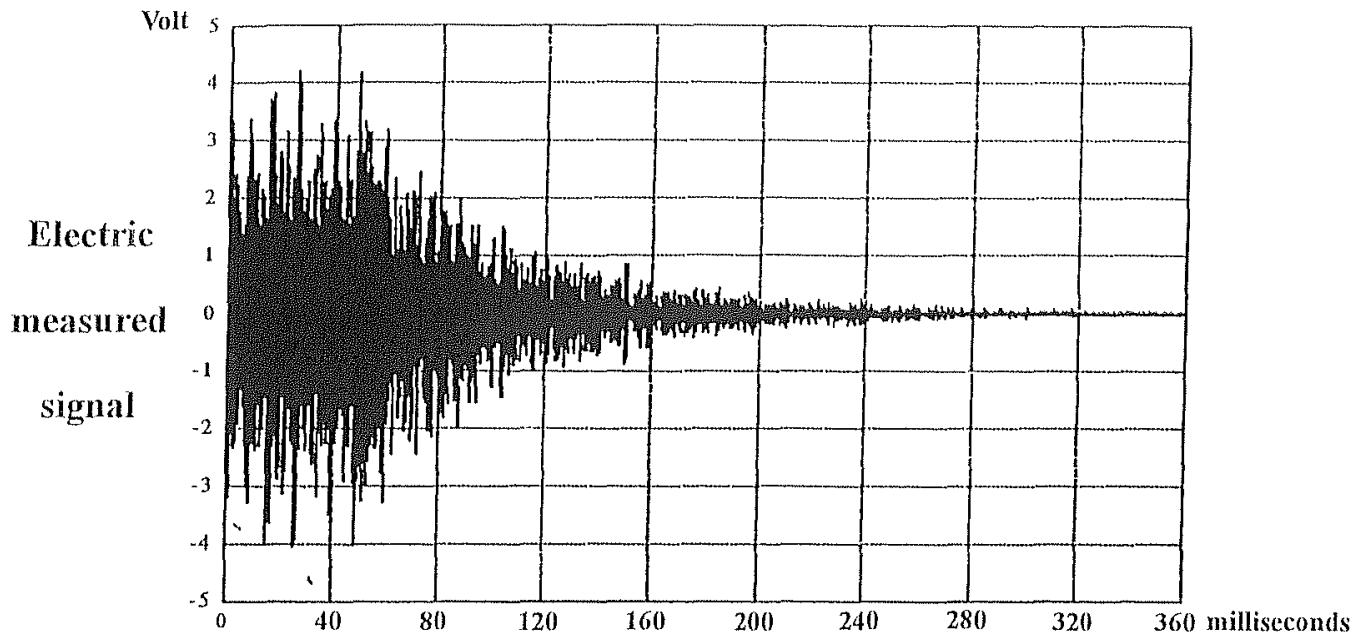

(a)

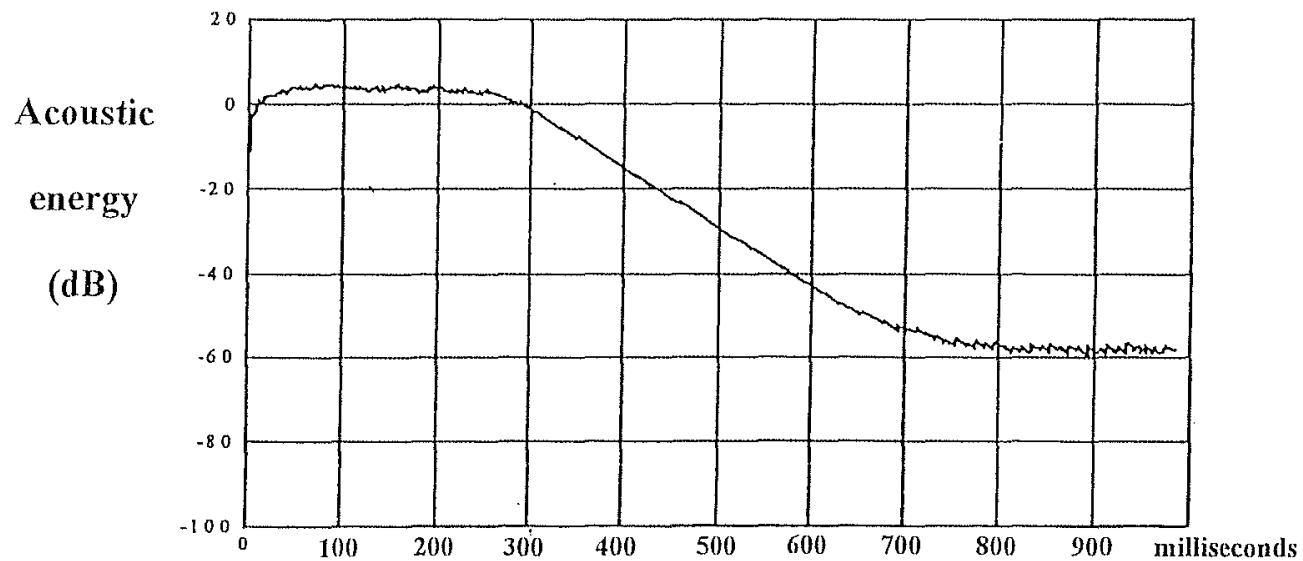

(b)

Fig. 2. Measurement of the reverberation time: (a) observed signal and (b) integrated signal power.

b) the calibration of the receiver $\left(S_{h}\right)$;

c) the measurement of the reverberation time;

d) the measurement of the mean square voltage.

In order to minimize the uncertainties a) we have to average the measurement on a sufficient number of modes. The number of modes averaged for the spectral pressure measurement is

$$
N_{m}=\frac{\Delta \nu}{\Delta f_{\text {rnean }}}=\frac{4 \pi f^{2} V \Delta \nu}{c^{3}}
$$

where $\Delta \nu$ is the frequency resolution of the spectral pressure and $\Delta f_{\text {mean }}$ the mean frequency separation between two modes given in $(10)$,

The measured spectral pressure is

$$
\hat{p}_{\text {rev }}(f)=p_{\text {rev }}(f)+\Delta p_{\text {rev }}(f)
$$

where $p_{\mathrm{rev}}(f)$ is the expected value and $\Delta p_{\mathrm{rev}}(f)$ the uncertainty. For the spectral pressure in $\mathrm{dB}$

$$
\begin{aligned}
10 \log \left[\hat{p}_{\mathrm{rev}}(f)\right] & =10 \log \left[p_{\mathrm{rev}}(f)+\Delta p_{\mathrm{rev}}(f)\right] \\
& \approx 10 \log \left[p_{\mathrm{rev}}(f)\right]+10 \log e \frac{\Delta p_{\mathrm{rev}}(f)}{p_{\mathrm{rev}}(f)} .
\end{aligned}
$$

The relative standard deviation is

$$
\frac{\Delta p_{\mathrm{rev}}(f)}{p_{\mathrm{rev}}(f)}=\frac{1}{\sqrt{N_{m}}}
$$

giving, in decibels, a standard deviation less than

$$
10 \log e \frac{1}{\sqrt{N_{m}}}=10 \log e \frac{\sqrt{c^{3}}}{f \sqrt{4 \pi V \Delta \nu}} .
$$

In the experiment $V=900 \mathrm{~m}^{3}$ and $\Delta \nu$ is greater than $100 \mathrm{~Hz}$. Finally the standard deviation of the spectral pressure in decibels is less than $237 / f$. Our frequency of interest is greater than 2 Khz. The uncertainties introduced by the interferences between the modes are thus less than $0.12 \mathrm{~dB}$.

The " $b$ " error is given by the caracteristics of the sensor. We use an omnidirectional standard hydrophone whose sensitivity does not depend on the acoustic wave field geometry.

The error " $c$ " and " $d$ " are statistical errors due to the integration finite time. Their values are fixed by the time-bandwidth product of the measurement.

The experimental results presented hereafter give a practical analysis of the accuracy.

\section{EXPERIMENTAL STUDY}

The experimental study has been done at IFREMER (Centre de Toulon-La Seyne) in a sea water tank of $10 \mathrm{~m} * 15 \mathrm{~m} * 6 \mathrm{~m}$. A schematic presentation of the experimental device is given in Fig. 1. The receiver is a Bruel\&Kjaer standard hydrophone 
(model 8100). This sensor is omnidirectional. An ITC 3013 projector is used for reverberation time measurement in order to have high source level to ensure the 60-dB dynamic giving a greater accuracy.

We first measure the reverberation time in different conditions and we estimate the direct field zone spreading. Then, using our procedure, we measure the source level of a calibrated source, in order to check the accuracy of our technique. Finally we measure the spectral level of the underwater vehicle Sirene.

\section{A. Reverberation Time Measurement}

We have conducted measurements on the complete bandwidth $[1 \mathrm{kHz}-20 \mathrm{kHz}]$ and some measurements on narrower band widths.

I) Broad-Band Reverberation Time: The transducer is driven with white noise in the frequency band [1 kHz-20 kHz]. Fig. 2 shows the different steps of measurement:

- the received signal after the switch off;

- the evolution of the received signal power measured with the integrator described in 3.1 with $\alpha=0.999$ giving an integration time of $48 \mathrm{~ms}$.

The values obtained for the reverberation time in different locations are given in Table I. We have quoted the standard deviation giving an experimental estimation of the precision.

The measurements in the tank give a value of $83.2 \mathrm{~m}^{2}$ for the Sabine coefficient. The surface of the reflective walls, excluding the air/water surface, is approximately $450 \mathrm{~m}^{2}$, giving a mean value of 0.82 for the reflexion coefficient of the walls.

The reverberation time varies when a device of great dimensions is immerged in the tank. The values of the reverberation time in presence of Sirene are given in Table II in different conditions of experiment. In the presence of Sirene, the Sabine coefficients has a mean value of $98.2 \mathrm{~m}^{2}$.

2) Reverberation in Narower Bands: We have measured the reverberation time in frequency bands of $2 \mathrm{kHz}$ centered on 8,10 , and $16 \mathrm{kHz}$. These measurements do not show a significant variation of the reverberation time with respect to central frequency.

\section{B. The Direct and the Reverberated Fields}

In order to determine experimentally the direct field zone and to verify the homogeneity of the reverberated field, we have made a spatial analysis of the acoustic field in the tank. Using a permanent emitting signal (wide-band, white noise), the acoustic energy clensity is plotted versus the distance to the source on a straight line originating at the emitter (Fig. 3).

In Fig. 3 we clearly see the direct field zone where the acoutic energy density quadratically decreases with distance and the reverberated field where the acoutic energy density is constant. This experiment gives two results. First, we estimate the direct field zone at $2.7 \mathrm{~m}$ and check that, thanks to the indetermination on the calculation of the direct field, it is coherent with the theoretical value $(1.8 \mathrm{~m})$ given by $(6)$ and the measured value of $T_{r}$. Then, we check the homogeneity of the reverberated field.
TABLE 1

REVERBERATION TIMES IN WIDE-BAND WITH A WhITE NOISE

\begin{tabular}{|c|c|}
\hline \multicolumn{2}{|c|}{$\begin{array}{l}\text { Spectral band } 1 \mathrm{Kl}-\mathrm{Iz}-20 \mathrm{kHz} \\
\text { Volume of the tank : } 900 \mathrm{~m} 3\end{array}$} \\
\hline Position & Reverberation time \\
\hline 1 & $406 \mathrm{~ms}$ \\
\hline 2 & $397 \mathrm{~ms}$ \\
\hline 3 & $396 \mathrm{~ms}$ \\
\hline 4 & $405 \mathrm{~ms}$ \\
\hline 5 & $397 \mathrm{~ms}$ \\
\hline 6 & $394 \mathrm{~ms}$ \\
\hline 7 & $401 \mathrm{~ms}$ \\
\hline 8 & $401 \mathrm{~ms}$ \\
\hline 9 & $393 \mathrm{~ms}$ \\
\hline Mean value & $399 \mathrm{~ms}$ \\
\hline Sd-deviation & $4.6 \mathrm{~ms}$ \\
\hline Precision & $1.2 \%$ \\
\hline
\end{tabular}

TABLE II

REVERBERATION TIMES IN WIDE-BAND WITH SIRENE

Reverberation time with SIRENE in the tank
$\begin{gathered}\text { Spectral band : } 1 \mathrm{kHz}-18 \\
\mathrm{kHz}\end{gathered}$
\begin{tabular}{c|c|c|c} 
Position & $\begin{array}{l}\text { Reverberation } \\
\text { time }\end{array}$ & Position & Reverberation time \\
\hline 1 & $346 \mathrm{~ms}$ & 1 & $346 \mathrm{~ms}$ \\
\hline 2 & $336 \mathrm{~ms}$ & 2 & $330 \mathrm{~ms}$ \\
\hline 3 & $337 \mathrm{~ms}$ & 3 & $339 \mathrm{~ms}$ \\
\hline 4 & $345 \mathrm{~ms}$ & 4 & $343 \mathrm{~ms}$ \\
\hline 5 & $341 \mathrm{~ms}$ & 5 & $336 \mathrm{mls}$ \\
\hline 6 & $332 \mathrm{~ms}$ & 6 & $327 \mathrm{~ms}$ \\
\hline 7 & $329 \mathrm{~ms}$ & 7 & $324 \mathrm{~ms}$ \\
\hline 8 & $335 \mathrm{~ms}$ & 8 & $331 \mathrm{~ms}$ \\
\hline mean & $338 \mathrm{~ms}$ & mean & $335 \mathrm{~ms}$ \\
\hline sd-dev & $6 \mathrm{~ms}$ & st-dev & $7 \mathrm{~ms}$ \\
\hline precision & $2 \%$ & precision & $2 \%$ \\
\hline
\end{tabular}

We can now proceed to the measurement of the spectral level. First, we use a calibrated source with a known spectral level; this, validates the method. Then we measure the spectral level of an operational source.

\section{Level of the Calibrated Source}

The source is an omnidirectional transducer B\&K 8105. The receiver is the B\&K 8100 hydrophone used previously. The distance $(5 \mathrm{~m})$ between the source and the hydrophone is greater than the limit of the direct field zone; we are in the reverberated field. We have conducted a series of experiments in wideband allowing, by filtering, to treat different kinds of bandwidth. Then we have used pure tones with several positions of the sensor, in order to verify the validity of the spatial averaging.

1) Wideband Measurement: The transducer is driven with a wideband white noise and the measurements are made versus frequency with a bandwidth of $100 \mathrm{~Hz}$. The spectra are averaged over $N$ segments of the signal. The relative standard deviation on the measured spectra is $1 / \sqrt{N}$. For the spectra measured, in decibels, the standard deviation is, using the same calculation as in $3.3,10 \log e / \sqrt{N} \mathrm{~dB}$. We average the spectra on $64 \mathrm{seg}-$ ments the relative standard deviation of the measured spectra 


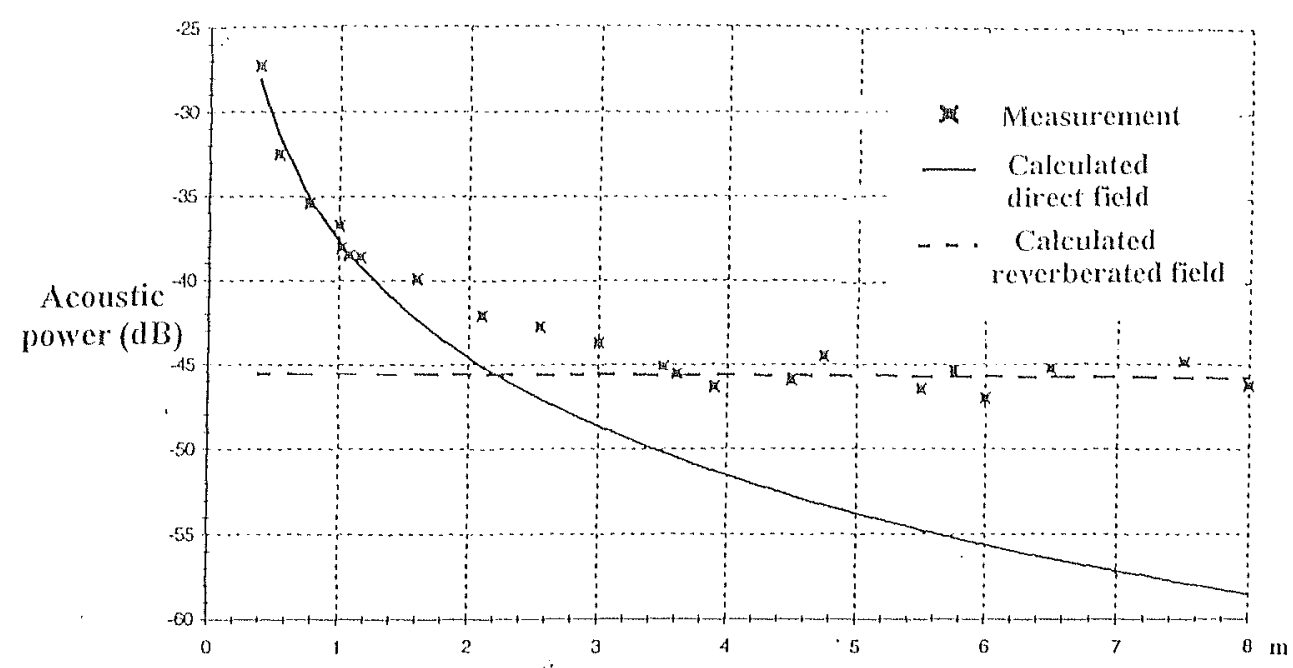

Distance from the source

Fig. 3. The acoustic energy density versus the distance to the souree.

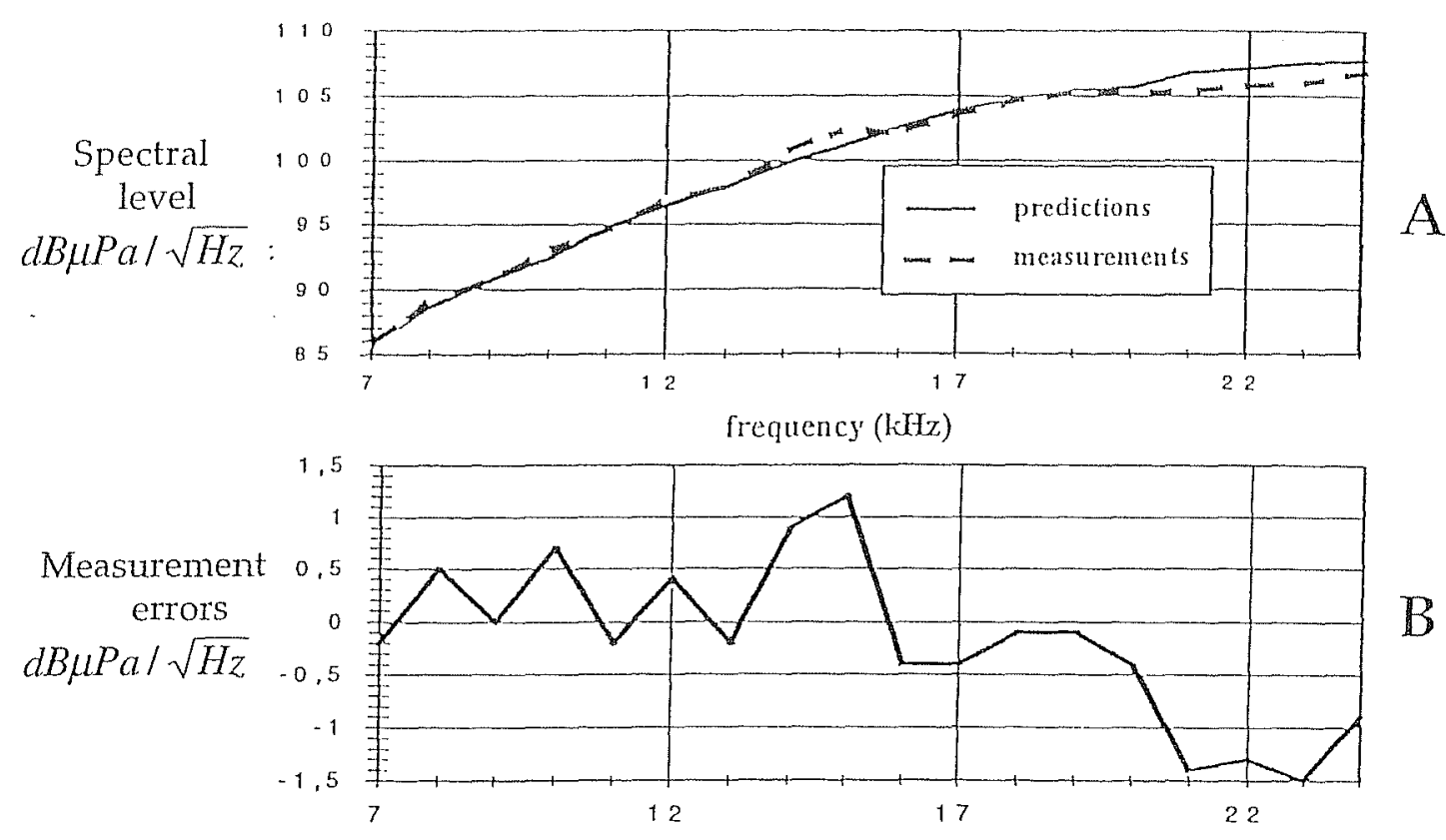

Fig. 4. Measurement of the source level. (a) Predicted and measured levels in decibels. (b) Difference between the predicted and measured levels in decibels.

(in decibels) is thus $0.5 \mathrm{~dB}$. The predicted values of the spectral level and the measured values are given on Fig. 4. In this figure, we have plotted the difference between the predicted spectral levels and the measured ones. This experiment shows that, for this source, we can reach a precision of $\pm 1.5 \mathrm{~dB}$ in the measurement of the spectral level.

2) Measurement with Pure Frequencies: Using the transducer B\&K 8105 we have emitted pure frequencies. With pure frequencies there is no mode mixing and the acoustic field is not constant in the reverberated field zone. The acoustic field oscillates around the reverberated field value.

We give in Table III the maximum, the minimum, and the mean value of the spatial acoustic field obtained at different frequencies. The mean values are in accordance with predicted values at $10 \mathrm{kHz}$ and $12 \mathrm{kHz}$, there is an unexplained discrepincy at $15 \mathrm{kHz}$.

\section{Measurement of Sirene NoIse Level}

Sirene is an experimental untethered unmanned underwater vehicle diving up to 6000 meter-depths connected by an acoustic link to the surface ( $\mathrm{Fig}$. 5). The measurement of the vehicle noise level has been done in the tank. The standard deviation, obtained in 44 experiments, is of $1 \mathrm{~dB}$.

We were not able to conduct an experiment on open sea using Sirene. Moreover, knowing that the experiments conducted on a calibrated source give a precision of $1.5 \mathrm{~dB}$ we can expect that this measurement technique should be able to give the level of an operational device with a precision of about $1.5 \mathrm{~dB}$.

\section{CONClusion}

Here we have given an experimental procedure for the measurement of the spectral level of a submarine acoustic source 
TABLE III

Maximum, Minimum AND MEAN VAlut: OF THE: LE:VEL. OF A PURE FREOUENCY SOURCE

\begin{tabular}{c|c|c|c|c|c}
\hline frequency & $\begin{array}{c}\text { maximum } \\
\mathrm{dB} \mathrm{V}\end{array}$ & $\begin{array}{c}\text { minimum } \\
\mathrm{dB} \mathrm{V}\end{array}$ & $\begin{array}{c}\text { mean } \\
\mathrm{dB} \mathrm{V}\end{array}$ & $\begin{array}{c}\text { mean measured } \\
\text { spectral level }\end{array}$ & $\begin{array}{c}\text { mean predicted } \\
\text { spectral level }\end{array}$ \\
\hline $10 \mathrm{kHL}$ & $-70,9$ & $-80,5$ & $-75,2$ & 135,1 & 135 \\
\hline $12 \mathrm{kHz}$ & $-68,5$ & $-81,2$ & $-74,5$ & 135,8 & 135,5 \\
\hline $15 \mathrm{kHz}$ & $-62,4$ & $-82,7$ & $-72,6$ & 138,7 & 144,5 \\
\hline
\end{tabular}

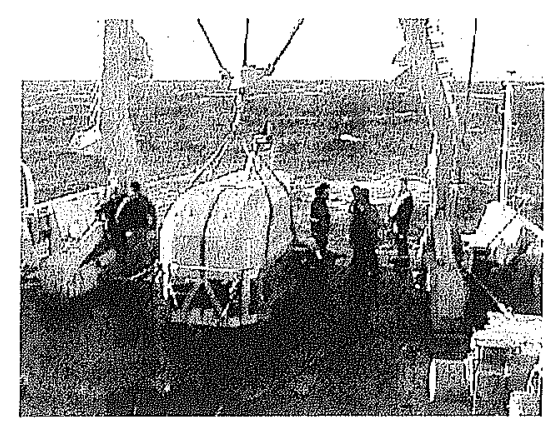

Fig. 5. Sirene schematic view.

in a tank. Although this procedure cannot give the directional properties of the source, it permits a measurement of the spatial mean spectral level with an accuracy experimentally evaluated at $\pm 1.5 \mathrm{~dB}$ using the transducer B\&K 8105 as source. In order to conclude definitively on the precision, experiments have to be done in order to compare the results given by our method and the results obtained in open sea for different sources.

Moreover, we think that this method of measurement, adapted from existing results in acrial acoustics and in electromagnetism, gives a useful tool for the characterization of the acoustic emission of the numerous devices operating in underwater environment.

\section{REFERENCES}

[1] W. S. Burdic, Underwater Aconstic System Analysis. Englewood Clitfs, NJ: Prentice Hall, 1984.

[2] J. Y. Chung and D. A. Blaser, "Transfer function method of measuring acoustic intensity in a duct system with flow," J. Acoust. Soc. Am., vol. 68, no. 6, pp. 1570-1577, Dec. 1980.

[3] A. R. Clark and P. S. Watkinson, "Measurement of underwater acoustic intensity in the nearfield of a point excited periodically ribber cylinder," in Shiphourd Acoustics, J. Buiter, Ed, Dordrecht, The Netherlands: Kluwer, 1986, pp. 177-188.

[4] P. Corona, G. Latmiral, and E. Paolini, "Use of a reverberating enclosure for measurements of radiated power in the microwave range," IEEE E.M. Comp., vol. 18, p. 54, 1976

[5] F. J. Fahy, Sound Intensity, Elsevier Applied Science. New York: Elsevier, 1989

[6] D. Hazelwood, "Measuring ROV noise in test tanks," in Underwater Svstem Design, July-Aug. 1996, pp. 10-14.

17] Y. Gabillet, "Etude playsique des salles réverbérantes et examen critique des normes de mesure de puissance acoustique et du coefficient d'absorption," Thèse de l'Université du Maine, Le Mans, 1980.
[8] H. Kultuff, Room Aconstics, 2nd ed: Applied Science Publishers, 1979:

[9] R. H. Lyon, "Statistical andlysis of power injection in structures and rooms," I. Acoust. Soc. Am., vol. 45, p. 545, 565, 1969.

[10] P. M. Morse and U. Ingard, Theoretical Acoustics. New York; McGraw Hill, 1968.

[11] W. C. Sabine, Collected Papers on Aconstics. Cambridge, MA: Harvall Univ. Press, 1927.

112| M. Schroeder, "Eigen-frequenzstatistik und anregungsstatistik in riumen," Acustica, vol. 4, p. 456, 1954.

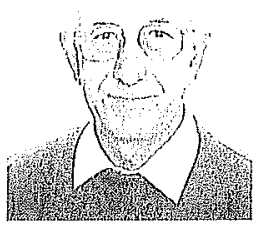

N. Cochard is a Graduate of the Electrician Engineers National Superior School of Grenoble (1996) and of the DEA on Signal Image and Speech at the National Polytechnic Institute of Grenoble (1996).

His research interests include signal, image, and speech processing with submarine acoustic measurements.

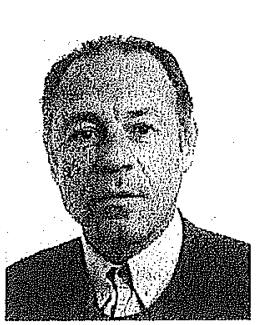

J. L. Lacoumewas born in Mirepoix, France in 1940 He is a graduate of the Ecole Normale Superienr (1964) and received the teaching certilicate (1964) and Ph.D. degree from the Paris University (1969).

He was a Lecturer at the University of Paris (1964-1972). Since 1972, he has been a Professor at the National Polytechnic Institute of Grenoble and Director of the laboratory of signal and image processing (LIS). His research interests are electromannetic waves in the ionosphere and magnetosphere, acoustic and elastic waves in the ocean and in the ground, and signal processing of spectral analysis, higher order statistics, and source separation.

Dr. Lacoume is a member of the French Society in Physics and the French Society on Electricity and Electronics. He received the Prix Hughes (1982) and Prix Charles Louis de Situlce de Freycinet (1990) of the French Academy of Sciences.

P. Arzeliès received the Ph.D. degree in acoustics in 1982.

He has been working at IFREMER since 1982, in charge of acoustic equipment and noise of deep civil submersibles, manned (NAUTILE) or unmanned (Side Scan Sonars, Remotely Operated Vehicles), as well as Assistant Manager of the Underwater Acoustic Laboratory.

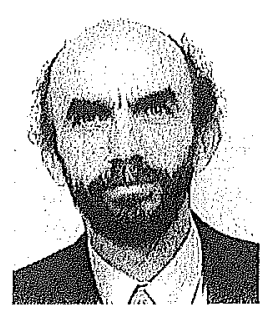

Y. Gabillet (deceased 1999) received the Ph.D. degree in acoustics from the University of Le Mans, France, in 1980

From 1981 to 1985 , he was consultant in acoustics. In 1985, he joined the Centre Scientificue et Technique de Batiment, CSTB, where he was involved in the research and development of outdoor noise propagation methods and softwares. From 1990 to 1991 , he was Guest Scientist of the National Research Council of Canada, Ottawal. 